\title{
INSEGNARE I LINGUAGGI SETTORIALI DI UNA LS A LIVELLO UNIVERSITARIO (IL CASO DELLA LINGUA ITALIANA). IL RUOLO E LE COMPETENZE DELL'INSEGNANTE E DELLO STUDENTE
}

\begin{abstract}
Il presente lavoro si propone di esaminare da un lato il ruolo e le competenze dell'insegnante di un corso di linguaggi settoriali mirato all'apprendimento e all'acquisizione di una dimensione specifica della lingua, ovvero per l'appunto il suo aspetto settoriale, e dall'altro, le competenze linguistiche attese negli apprendenti di tale corso. Entrambe le posizioni saranno analizzate in base al contesto di studio quello universitario - e agli obiettivi didattici dettati da tale contesto.

Riguardo alle competenze dell'insegnante, esamineremo due opinioni suggerite dalla letteratura sull'argomento (Gotti, 1992: 286-289): a) l'insegnante deve possedere, oltre ad ottime conoscenze linguistiche, anche nozioni specifiche del settore; b) nel caso in cui l'insegnante possedesse prevalentemente solo elevate competenze linguistiche, dovrebbe applicare una didattica che, attraverso varie attività comunicative, avrebbe come scopo lo scambio di conoscenze non-linguistiche con lo studente. In questo caso, entrambi i partecipanti al processo didattico saranno in grado di analizzare e comprendere l'aspetto contenutistico del materiale.

Lo sviluppo e lo stato delle competenze linguistiche attese negli apprendenti sarà esaminato nell'ottica del menzionato contesto di studio e in stretto collegamento con i bisogni degli studenti stessi. Sarà presentata la struttura didattica del corso Linguaggi settoriali dell'italiano, tenutosi nel primo semestre dell'a.a. 2017/2018 presso il Dipartimento d'italianistica della Facoltà di Filologia a Belgrado. I partecipanti sono stati gli studenti d'italianistica e il corso prevedeva, oltre alla parte teorica dedicata alla presentazione di diverse peculiarità linguistiche (morfologiche, sintattiche, semantiche e lessicali) dei vari linguaggi settoriali (economia, religione, politica, legge), anche attività di analisi e di traduzione dei rispettivi testi.
\end{abstract}

Parole chiave: linguaggi settoriali, lingua italiana, competenze, insegnante, apprendente 
Jelena R. Drljević

\section{Introduzione}

Nel primo capitolo, accanto ad una breve definizione dei linguaggi settoriali (LS) e delle loro particolarità linguistiche, ci dedicheremo alla questione delle competenze reali e di quelle effettivamente richieste all'insegnante e allo studente che si avventurano, ciascuno dal suo canto, nello studio di un linguaggio settoriale. Presenteremo le opinioni scientifiche rilevanti e i vari punti di vista che emergono nella letteratura sull'argomento.

Nell'ambito della descrizione del corso Linguaggi settoriali dell'italiano che ogni anno si svolge presso il Dipartimento d'italianistica della Facoltà di Filologia a Belgrado, esamineremo in particolar modo gli approcci e i metodi glottodidattici messi in atto dall'insegnante nel gestire il citato corso. D'altra parte, prenderemo in esame le preconoscenze linguistiche generali, nonché i futuri bisogni linguistici e professionali degli studenti, laureati in lingua e letteratura italiana. Presenteremo anche i dati rilevati dal questionario distribuitogli alla fine del corso.

La parte conclusiva ci servirà a definire meglio i punti salienti inerenti alle competenze richieste all'insegnante di un corso di linguaggi settoriali, ma anche ai bisogni e alle competenze dei suoi studenti. In base a tali conoscenze delineeremo alcune modifiche e/o cambiamenti del corso menzionato, allo scopo di migliorare l'approccio didattico globale e i suoi esiti finali.

1. Definizione della nozione di linguaggi settoriali e principali peculiarità linguistiche

La nozione linguaggi settoriali ${ }^{1}$ trova le sue origini e la sua continua evoluzione nello sviluppo e settorializzazione di molteplici campi occupazionali dell'uomo. Si tratta di lingue che sono "legate a particolari attività lavorative e professionali" (Sobrero, 1993: 237). Come sottolinea Berruto (1993: 9) questo codice linguistico può essere definito anche come la varietà diafasica di una lingua, dato che consente la comunicazione tra gli specialisti di un certo campo, in una specifica situazione o contesto d'uso. Si mette in rilievo, dunque, il suo uso prevalentemente pragmatico in contesti specifici di natura scientifico-professionale (Balboni, 2000: 10).

1 Sulle diverse denominazioni del campo sviluppate nella lingua italiana consultare Balboni 2000, Borello 1994, Beccaria 1973, Berruto 1987, Sobrero 1993. 
A differenza della lingua comune, questa lingua fa uso di un particolare discorso, nonché di un lessico e di strutture grammaticali a sé stanti. In quanto alla struttura sintattica dei testi specialistici generalmente sono riscontrabili le seguenti caratteristiche: a) è presente una struttura sintattica compatta e sintetica che in molti casi deriva dall'omissione degli articoli, specialmente nei testi di natura tecnica o burocratico-giuridica (Gotti, 1992: 208); b) a questa semplificazione della sintassi contribuisce in larga misura l'uso dello stile nominale che riduce l'importanza del verbo, limitandone i tempi, i modi, la quantità e la ricchezza semantica (Biagi, 1990: 341-348); c) di frequente si ricorre a forme impersonali e passive, il cui uso è particolarmente evidente nei testi scientifici (Sobrero, 1993: 250 251); d) fatta eccezione per il linguaggio giuridico e quello burocratico, in cui vengono utilizzate prevalentemente proposizioni subordinate, è dominante, a livello sintattico, una chiara tendenza ad economizzare l'enunciato, utilizzando, a questo scopo, il rapporto di coordinazione tra le proposizioni (Balboni, 2000: 40; Samardžić, 2006: 45).

Una delle proprietà maggiormente espresse nei linguaggi settoriali è proprio il lessico. Un termine tecnico o scientifico, come osservano molti autori (Gotti, 1992: 240-256; Sobrero, 1993: 244-246; Balboni, 2000: 4552), contiene alcune caratteristiche differenti rispetto alle parole comuni: a) forte monoreferenzialità che, in un determinato contesto disciplinare, riduce l'aspetto connotativo dell'unità lessicale; b) precisione e trasparenza che consentono un immediato riconoscimento del termine, specialmente tra i membri di un settore specialistico. Dal punto di vista lessicologico, si riscontra una forte produttività nella formazione di termini specialistici. Un notevole numero di termini proviene da processi di derivazione, il che determina l'esistenza di numerosissimi prefissati (anticolesterolo, bicamerale), suffissati (bronchite, fibrillazione), ma anche forme verbali parasintetiche (revitalizzazione). I termini derivano anche da processi di composizione (aortocoronarico, vasodilatatore), nell'ambito della quale si riscontrano moltissimi casi di forme non libere (monocamerale, tomografia). Un numero rilevante di lessemi è costituito dai prestiti, tra i quali si registra un largo uso di prestiti di necessità (hi-tech, smokey eyes), prestiti di lusso (download), calchi semantici (sito, aggiornare) e calchi traduzione (disco rigido, salvaschermo).

Pur avendo tra le sue caratteristiche linguistiche numerosi aspetti particolari, molti studiosi ritengono che questa lingua rappresenti solamente 
Jelena R. Drljević

uno dei modi in cui la lingua comune può essere usata (Bugarski, 1997: 201). Prendendo in considerazione tutto quello che abbiamo detto finora sui linguaggi settoriali, possiamo concludere che questa varietà linguistica fa parte della lingua generale, ma ne usa gli elementi e le categorie per scopi specifici, con modalità particolari e con frequenza e distribuzione diverse da quelle della lingua comune (Gotti, 1992: 207; Đorović, 2011: 752-753).

\subsection{Ruolo e competenze dell'insegnante di un corso} sui linguaggi settoriali

Nell'esaminare le competenze di un insegnante, partiamo dai due presupposti suggeriti da Gotti (1992: 283-289): a) l'insegnante deve possedere, oltre ad ottime conoscenze linguistiche, comprese quelle inerenti alle peculiarità strutturali e terminologiche (vedi 1), anche quelle relative alle nozioni e ai concetti specifici del settore scientificoprofessionale; nel caso in cui l'insegnante possedesse prevalentemente solo elevate competenze linguistiche, dovrebbe applicare una didattica che, attraverso varie attività comunicative nella rispettiva lingua straniera, favorirà lo scambio di conoscenze non-linguistiche con lo studente. In questo caso, entrambi i partecipanti del processo didattico saranno in grado di analizzare e comprendere l'aspetto contenutistico del materiale.

Nell'analizzare più da vicino queste due ipotesi, è opportuno fare un breve accenno ad alcuni autori, esperti che hanno lavorato nell'ambito dei primi studi scientifici sui linguaggi settoriali - studi che si rivolgevano, in un primo momento, quasi esclusivamente alla lingua inglese e all'ampio spettro delle ricerche su English for Specific Purposes (ESP). Ricordiamo Strevens (1980: 119) che ha definito l'insegnante di un corso sui linguaggi settoriali "superior teacher" ed Ewer (1983: 9-31) che nell'ambito della ricerca generale sui linguaggi settoriali si è dedicato particolarmente alla questione della formazione degli insegnanti, prestando particolare attenzione ai loro bisogni, alle competenze e ai ruoli. Quest'autore, tra le varie problematiche riguardanti i requisiti dell'insegnante, riporta anche quella inerente alla mancata conoscenza di concetti specifici di natura scientifico-professionale.

Nel maggior numero dei casi l'istruzione dell'insegnante di un corso sui linguaggi settoriali è fondata sulla conoscenza di nozioni appartenenti alle scienze umanistiche. Generalmente gli insegnanti 
provengono da un contesto di studio universitario delle lingue straniere e si specializzano ulteriormente in vari campi interdisciplinari, ma comunque appartenenti all'area umanistica, quali sociolinguistica, pedagogia, scienze della comunicazione e simili. Ne deriva che l'insegnante di un corso sui linguaggi settoriali, specialmente all'inizio della sua carriera, probabilmente non possederà, o possederà solo in misura molto ridotta, conoscenze strettamente disciplinari e relative ad ambiti rigorosamente tecnici. A nostro avviso, anche se a prima vista questo aspetto costituisce il maggiore ostacolo, vanno comunque presi in considerazione molteplici fattori: il livello di conoscenza degli specifici contenuti settoriali, la scelta del materiale che sarà usato in classe, l'analisi dei bisogni degli allievi, gli obiettivi prescritti dal sillabo, nonché, in fin dei conti, la volontà dell'insegnante di acquisire nuove conoscenze, almeno quelle di base, che lo aiuteranno nello svolgimento del lavoro.

Il ruolo dell'insegnante di un corso sui linguaggi settoriali si estende in base alle molteplici esigenze del processo dell'insegnamento e così gli autori Dudley-Evans e St John (1998: 13-17), avendo in mente, in primo luogo, gli insegnanti dell'ESP, preferiscono il termine "practitioner" (professionista) al posto di "teacher" (insegnante). Secondo questi due autori il/la professionista di un corso sui linguaggi settoriali ricopre $\mathrm{i}$ seguenti ruoli:

Il professionista come insegnante spesso si troverà in contesti d'insegnamento diversi, in cui sarà richiesta la massima flessibilità e la capacità di adattarsi a ruoli differenti. Nelle classi in cui l'insegnante si troverà di fronte a studenti che hanno una conoscenza dei contenuti specifici migliore della sua, la strategia sarà di indirizzare la dinamica del lavoro sulle competenze comunicative, più che sugli aspetti contenutistici. D'altra parte, può succedere che l'insegnante assuma il ruolo di tutor, nei casi in cui dovrà dare dei consigli inerenti alla scrittura e alla struttura discorsiva delle tesi o degli articoli studenteschi scientifici che saranno pubblicati nelle riviste internazionali. Dunque, l'insegnante per un verso sarà ascoltatore, interessato a capire e imparare i contenuti specifici che sono oggetto di studio dei suoi studenti, d'altra parte si assumerà la responsabilità di guidare la classe, rispettando gli obiettivi del corso (Dudley-Evans e St John, 1998: 13-14).

Il professionista come ideatore del corso e fornitore del materiale. Nel maggior numero dei casi l'insegnante dovrà provvedere a selezionare, 
Jelena R. Drljević

oltre al libro di classe - nel caso esista - anche del materiale supplementare, adeguato ai bisogni specifici degli studenti e che si attenga agli obiettivi del corso stesso (idem, 14-15).

Il ruolo del ricercatore copre molteplici aspetti del lavoro dell'insegnante-professionista di un corso sui linguaggi settoriali. È orientato maggiormente sulla progettazione del corso, con particolare attenzione agli obiettivi ed esiti finali richiesti agli studenti, alla scelta e all'analisi del materiale usato in classe, all'analisi e all'individuazione dei bisogni degli studenti (Đorović, 2015). I dati della sua ricerca andrebbero, in un secondo momento, implementati nelle attività didattiche e aggiornati in base alle future indagini del campo d'interesse o agli eventuali cambiamenti relativi ai bisogni degli studenti (idem, 15).

Il professionista come collaboratore - questo ruolo dell'insegnante consisterebbe, nel caso di un'organizzazione ideale del corso, in una sua collaborazione con i veri professionisti del campo scientifico-professionale. In questo reciproco scambio di esperienze, gli esperti provvederebbero a fornire un'approfondita analisi del testo dal punto di vista del contenuto specifico mentre l'insegnante, avvalendosi del loro aiuto, potrebbe integrare tali conoscenze nel suo programma e nelle attività linguistiche del corso (idem, 15-16). Quello di insegnare i contenuti specifici è propriamente compito degli esperti, e non rientra nelle competenze dell'insegnante di lingua (Gotti, 1998: 232).

Il compito della verifica e della valutazione di un corso, nonché delle conoscenze acquisite dagli studenti, rappresenta uno dei momenti di maggiore complessità in qualsiasi percorso didattico. Questo passo prevede una serie di osservazioni, che, applicate ad un corso sui linguaggi settoriali, potrebbero essere riassunte nel seguente modo: a) il momento di valutazione dell'andamento del corso, ovvero la valutazione del livello di corrispondenza tra i bisogni degli studenti e gli obiettivi del corso, la valutazione del materiale, delle tempistiche nell'ambito del programma ecc; b) la questione relativa ai tipi di materiale (testo) adoperato nelle prove finali di conoscenza della lingua (tre sono opinioni al riguardo: il testo dovrebbe essere generale; il testo dovrebbe essere autentico, specialistico; il materiale deve rappresentare l'integrazione dei due aspetti); c) il momento della verifica delle conoscenze degli studenti, che devono riguardare, senza dubbio, le conoscenze linguistiche e non quelle del contenuto (Gotti, 1992: 270); d) il momento della decisione sui criteri di valutazione della prova e 
INSEGNARE I LINGUAGGI SETTORIALI DI UNA LS A LIVELLO ...

costruzione di un sistema corretto e sostenibile di assegnazione dei valori (Gotti, 1992: 274-282, Gotti, 1998: 227-245) ${ }^{2}$.

Nel cercare di giungere ad una conclusione quanto più globale possibile sul ruolo e sulle competenze dell'insegnante di un corso sui linguaggi settoriali, ci avvaliamo delle considerazioni di Gotti (1992: 294): "l'insegnante di linguaggi settoriali è un linguista che analizza il linguaggio scientifico (...), usando la metodologia dell'analisi linguistica e disciplinare". Dunque, l'insegnante possiede, grazie alla sua formazione linguistica, ma anche a quella didattica, le conoscenze necessarie per poter svolgere un'analisi approfondita della struttura testuale e di quella microlinguistica. Parallelamente con questo lavoro, di cui è esperto e che rientra nelle sue specifiche competenze, dovrebbe essere, secondo la nostra opinione, interessato a conoscere anche contenuti nonlinguistici, consultando, se possibile, specialisti dei vari settori scientificoprofessionali.

\subsection{Ruolo e competenze dello studente di un corso} sui linguaggi settoriali

Per quanto concerne le competenze e il ruolo dello studente di linguaggi settoriali, questo aspetto è strettamente legato alla questione dell'analisi dei suoi bisogni. Non è nostra intenzione, in questo contributo, affrontare in modo preciso e dettagliato la complessa questione relativa all'analisi dei bisogni, dalla quale dipende, tra l'altro, tutta la progettazione curricolare di un corso scolastico. Bisogna ricordare, però, che nella progettazione di un corso sui linguaggi settoriali i bisogni degli studenti sono facilmente identificabili, e così anche gli obiettivi stessi del corso, perché si ha in mente una situazione specifica, basata sulle circostanze della vita reale, in cui una certa lingua si usa o sarà usata (Đorović, 2015: 22).

Prendendo spunto da alcuni approcci teorici e dai rispettivi modelli che si occupano dell'analisi dei bisogni, come per esempio quello sociolinguistico di Munby (1978) e la versione integrata di Tarone e Jule (1989), il modello sistematico di Richterich e Chancerel (1977) che si basa sui sillabi nozional-funzionali di Wilkins (1976), quello di Hutchinson

Sui diversi spunti di riflessione, nonché sulle esigenze oggettive e scientificamente giustificate per l'esistenza di una verifica e valutazione delle conoscenze dei linguaggi settoriali consultare Douglas, D. (2000). Assessing Languages for Specific Purposes. Cambridge University Press. 
e Waters (1987) che in linea molto generale si basa sulle modalità d'apprendimento degli studenti e altri ${ }^{3}$, e prendendo in considerazione gli obiettivi principali di un corso sui linguaggi settoriali definiti da Gotti (1992: 37-38), cercheremo ora di elencare le conoscenze necessarie per uno studente. Lo studente di un corso sui linguaggi settoriali, in base alle esigenze particolari del corso e all'analisi approfondita dei bisogni, deve sviluppare i seguenti aspetti linguistici (grammaticali) e comunicativi:

Abilità linguistiche sia di comprensione che di produzione scritta $\mathrm{e}$ orale, con speciale riguardo alla stesura dei testi richiesti nei particolari contesti d'uso (lettere, relazioni ecc).

Competenze in traduzione dei testi di carattere scientificoprofessionale.

Abilità di analisi morfosintattica e testuale dei testi di carattere scientifico-professionale.

Conoscenze di vari aspetti semantico-lessicali, quali la formazione delle parole, nonché le regole di funzionamento dei termini specifici.

Conoscenze non-linguistiche, appunto quelle concettuali e contenutistiche di un certo campo, il cui livello dipende dalle esigenze dei contesti d'uso.

Nel capitolo che segue presenteremo la struttura del corso Linguaggi settoriali, tenutosi nell'a.a. 2017/2018 alla Facoltà di Filologia a Belgrado, il suo programma didattico, l'approccio e i metodi usati in classe, con particolare attenzione al ruolo dell'insegnante e alle sue competenze, nonché a quelle degli studenti. Riporteremo, inoltre, i risultati del questionario compilato dagli studenti, mettendo in rilievo tutte le peculiari caratteristiche di questo gruppo di utenti.

2. Il Corso Linguaggi settoriali dell'italiano presso il Dipartimento d'italianistica della Facoltà di Filologia a Belgrado

\subsection{Obiettivi del corso}

L'obiettivo principale del corso è stato quello di avvicinare gli studenti ai linguaggi settoriali, alla loro struttura linguistica, all'organizzazione testuale, alle modalità di funzionamento e alle regole di traduzione.

3 Per una rassegna più completa e dettagliatamente esaminata consultare Đorović (2015: 31-42). 
INSEGNARE I LINGUAGGI SETTORIALI DI UNA LS A LIVELLO ...

Inoltre, agli studenti è stata data la possibilità di applicare le preconoscenze morfosintattiche sull'analisi di testi tecnici, di esercitarsi nell'esposizione orale di tale analisi, di creare glossari di termini tecnici che avrebbero rappresentato il punto di partenza per successivi approfondimenti e ampliamenti del lessico settoriale, ma anche di avere una prima visione sui contenuti non strettamente linguistici. Dunque, il corso sui linguaggi settoriali si è proposto come introduzione ad una varietà linguistica poco o affatto conosciuta dagli studenti - un primo incontro volto a favorire le capacità di autoapprendimento, indispensabili sia per l'inserimento nel mercato del lavoro che per il futuro perfezionamento professionale.

\subsection{Struttura del corso}

Il corso Linguaggi settoriali prevede nel primo semestre due mesi di lezioni teoriche e pratiche della durata complessiva di 16 ore in classe e la possibilità di incontrare le insegnanti due volte alla settimana durante le ore di ricevimento. Nel secondo semestre, per gli studenti interessati a questo argomento, è prevista la stesura della tesi di master. Nell'a.a. 2017/2018 la struttura del corso ha previsto i seguenti segmenti e attività:

Introduzione teorica

- la situazione sociolinguistica in Italia e le varietà del repertorio linguistico italiano;

- la presentazione della definizione e delle proprietà funzionali e linguistiche dei linguaggi settoriali;

- la presentazione dei campi professionali sui quali è incentrata l'analisi linguistica e la traduzione: economia, industria elettromeccanica, energetica, industria chimica e petrolifera, telecomunicazioni e informatica, istituzioni dell'UE, interpretariato e traduzione nell'UE, industria militare, finanze e sistema fiscale, istituzioni finanziarie internazionali, partiti politici e ordinamento statale, agricoltura e industria alimentare, diritti dell'uomo, ONG, industria della bellezza, previdenza sociale, commercio e marketing, istruzione, cronaca nera, criminalità;

- la presentazione dell'approccio testuale alla traduzione di un testo scientifico-professionale (fasi della traduzione: lettura, traduzione, rilettura). 
Jelena R. Drljević

Attività 1: lavoro in gruppo

Si esegue in gruppi di 2 o 3 studenti che hanno il compito di trovare un testo in italiano attinente al campo professionale precedentemente scelto. Il testo deve essere lungo 1000 parole, reperibile su Internet, su un quotidiano o in qualche rivista di stile scientifico popolare. Il testo, dopo esser stato visionato e approvato dall'insegnante, viene tradotto in serbo e gli studenti ne fanno una presentazione orale contenente l'analisi morfologica, sintattica e lessicale. Oltre all'analisi linguistica devono essere esposti i maggiori problemi di traduzione. In base al testo tradotto i gruppi devono creare anche un glossario di termini tecnici individuati nel testo con la possibilità di ampliare la lista aggiungendo altri vocaboli degli stessi campi semantici.

Attività 2: traduzione individuale

Traduzione di un testo specializzato scelto dall'insegnante. L'attività si svolge in classe.

\section{Attività 3: traduzione per la NURDOR ${ }^{4}$}

L'attività si svolge individualmente. L'insegnante divide il testo da tradurre ${ }^{5}$ tra gli studenti e il processo traduttivo si esegue con il sostegno degli insegnanti di lingua e di specialisti del settore (psicologi, pediatrici, oncologi) ${ }^{6}$.

Attività 4: stesura di composizione scritta

Comprende la stesura di una composizione di 15000 caratteri basata su un corpus di 3 testi in italiano appartenenti a un campo settoriale diverso da quello analizzato nell'ambito della prima attività. I testi devono essere analizzati, commentati e tradotti in serbo e ne deve conseguire un glossario.

4 NURDOR - Nacionalno Udruženje Roditelja Dece Obolele od Raka (Associazione nazionale dei genitori di bambini malati di cancro).

5 I testi appartengono al campo della medicina e della psicologia con particolare riguardo alla psicooncologia pediatrica. I testi vengono tradotti dall'italiano al serbo.

6 Finora sono stati pubblicati i seguenti libri: Scarponi, D. (2012). Svaki trenutak je važan. Razmišljanja iz oblasti pedijatrijske psihoonkologije. Beograd: NURDOR; Scarponi, D., Pession, A. (2013). Specifičan bol u detinjstvu, za razumevanje patnje u pedijatriji. Beograd: Nurdor; Natalicchio, P. (2017). Kraljevstvo PO. Beograd: Nurdor. E' in fase di preparazione per la stampa la traduzione eseguita nel 2017 - 2018. 
INSEGNARE I LINGUAGGI SETTORIALI DI UNA LS A LIVELLO ...

2.3. Ruolo, competenze e approccio didattico dell'insegnante del corso Linguaggi settoriali dell'italiano

Come è stato già accennato nella parte 1.1 , l'insegnante di lingua straniera coinvolto nell'insegnamento dei linguaggi settoriali in molti casi non predispone di conoscenze in campi non linguistici.

Nel nostro caso, nonostante una formazione linguistica e una specializzazione nel campo della didattica delle lingue straniere (più strettamente nello sviluppo della competenza lessicale di una lingua straniera), grazie ad una notevole esperienza nell'ambito della traduzione ed interpretariato possedevamo le conoscenze di base di alcuni concetti appartenenti a settori quali medicina, economia, giurisprudenza, tutela dell'ambiente. Queste conoscenze ci hanno aiutato a svolgere meglio e con più facilità il lavoro primario, ovvero ad assumere i vari ruoli elencati nella parte 1.1 .

Per quanto invece riguarda la gestione delle conoscenze concettuali di natura scientifico-professionale, il ruolo ricoperto in classe è stato orientato verso una collaborazione e uno scambio di conoscenze con gli studenti. Su questo percorso abbiamo condiviso con gli studenti la curiosità verso la comprensione dei contenuti specializzati, scoprendo insieme nuovi orizzonti del sapere umano. In un clima di reciproca motivazione, gli studenti hanno dimostrato particolare interesse per i settori sempre attuali, quali economia e giurisprudenza.

Sul piano strettamente linguistico, abbiamo scelto l'approccio suggerito da Mazzotta (2007: 12-23). L'autrice propone un ordine didattico che preveda in un primo momento attività di comprensione scritta durante le quali lo studente cerca di capire e interiorizzare l'argomento e i concetti del settore scientifico-professionale. Questo processo metacognitivo, nella fase successiva, andrà accompagnato da una riflessione metalinguistica, nella quale si esegue l'analisi morfosintattica e lessicale. Prima di passare alla fase produttiva, ovvero alla traduzione del testo, l'autrice propone una fase facoltativa che ha come scopo la traduzione orale per singoli brani. Questo processo graduale ovviamente non finisce con la resa del testo tradotto nella L1 o nella LS, ma richiede ulteriori e accurate riletture nonché gli aggiustamenti necessari a rendere il testo d'arrivo scorrevole sia dal punto di vista linguistico che da quello contenutistico. 
Jelena R. Drljević

\subsection{Ruolo e competenze degli studenti del corso Linguaggi} settoriali dell'italiano

Il corso è stato attivato con degli studenti già laureati che, avendo acquisito elevate conoscenze sul funzionamento della lingua comune, erano pronti a sviluppare delle conoscenze linguistiche aggiuntive e quelle appartenenti a campi non strettamente linguistici.

Gli studenti universitari di lingue e letterature straniere rappresentano un gruppo particolare nell'ottica dell'insegnamento e dell'apprendimento dei linguaggi settoriali. A differenza di tutti gli altri studenti, che nel corso degli studi gradualmente acquisiscono le conoscenze di vari settori occupazionali, lo studente di lingue e letterature straniere di solito dispone delle conoscenze dei principali campi d'indagine delle scienze umane mediante gli apporti specifici e interdisciplinari della cultura pedagogica, psicologica e sociolinguistica. Il suo punto forte sta prima di tutto nel possesso di approfondite conoscenze e competenze linguisticocomunicative di cui può servirsi nell'analizzare e apprendere i linguaggi per scopi specifici (Drljević, 2016: 497).

Uno studente del Dipartimento d'italianistica della Facoltà di Filologia a Belgrado struttura il suo percorso formativo in un contesto specifico. Il programma quadriennale d'insegnamento di lingua italiana alla Facoltà di Filologia non prevede l'apprendimento dei linguaggi settoriali, ma si incentra sull'acquisizione delle discipline teoriche (fonetica e fonologia, lessicologia, morfologia, semantica, sintassi, grammatica storica, analisi contrastiva ecc), sullo sviluppo delle abilità linguistiche, nonché sul raggiungimento di adeguate competenze sociolinguistiche e pragmatiche. Dopo gli studi quadriennali, lo studente dispone di conoscenze stabili ed approfondite sul funzionamento complessivo del sistema linguistico. Anche se la didattica dell'insegnamento delle LS nell'ambito degli studi filologici è storicamente basata sulle discipline teoriche di ordine linguistico e letterario, dall'introduzione dei principi di Bologna essa si è aperta a nuovi programmi di natura interdisciplinare. Questa nuova prospettiva ci ha spinto ad attivare un corso che aiuti gli studenti ad arricchire sia il loro repertorio linguistico che la visione su possibili domini e situazioni nei quali una lingua specializzata potrà essere usata (Drljević, 2016: 496).

Riguardo alla generazione degli studenti che nell'a.a. 2017/2018 hanno frequentato il corso Linguaggi settoriali dell'italiano, non abbiamo riscontrato particolari problemi nell'andamento del corso. Benché 
non possedessero sviluppate conoscenze settoriali, gli studenti hanno dimostrato un notevole interesse per il materiale presentatogli in classe e una competenza stabile nell'applicare le conoscenze linguistiche, acquisite nel percorso quadriennale degli studi, in attività di comprensione, analisi e traduzione dei testi specializzati. Inoltre, il gruppo si è attivamente impegnato (insieme all'insegnante) nei lavori di ricerca sui contenuti, sulla terminologia, nonché sulle soluzioni traduttive dei testi.

\subsubsection{I risultati del questionario.}

Dopo il corso abbiamo distribuito agli studenti un questionario di valutazione, con l'intenzione di scoprire i punti forti e quelli deboli del corso, il livello di soddisfazione degli studenti, nonché di esaminare l'adeguatezza della struttura e del programma offerto. Il questionario consiste di una nota introduttiva e di una serie di domande sul corso. Le domande sono, in generale, strutturate sul modello di scelta multipla, tranne una domanda aperta. Il questionario è anonimo ${ }^{7}$.

Nel raccogliere i risultati abbiamo scoperto che le risposte delle dieci studentesse che hanno partecipato al corso sono quasi identiche. L'unica vera differenza riguarda la domanda numero 2.

Dunque, in base alle risposte alla domanda numero 1, abbiamo scoperto che le studentesse, prima di iscriversi al corso Linguaggi settoriali dell'italiano, avevano poca esperienza con la lettura, l'analisi e la traduzione dei testi appartenenti ad un settore scientifico-professionale. La loro esperienza consisteva, prevalentemente, nella lettura di testi del genere specializzato, con nessun'esperienza di traduzione di tali testi.

Con la domanda successiva abbiamo voluto scoprire i motivi dell'iscrizione al corso. Riteniamo che le risposte siano di notevole importanza, perché ci aiutano a individuare le tendenze degli studenti d'Italianistica dell'Università di Belgrado dopo il conseguimento della laurea in lingua e letteratura italiana. In un certo senso, grazie a queste risposte siamo diventati più consapevoli di quelli che gli studenti stessi considerano come i loro punti deboli. Per il 50\% delle intervistate lo sviluppo delle competenze traduttive nell'ambito della traduzione dei testi specifici rappresenta il motivo principale per l'iscrizione al corso. Per due studentesse il corso è stato una buona opportunità per migliorare le

7 Il questionario si trova nell'allegato. 
Jelena R. Drljević

conoscenze generali linguistico-comunicative della lingua italiana, mentre tre di loro ritengono che il corso sia stato utile per il consolidamento delle conoscenze sia linguistiche che non-linguistiche.

Il 90\% di loro ha seguito senza problemi la struttura e il contenuto del corso, mentre solo una studentessa ha riscontrato problemi nella terminologia dei testi, considerandola troppo difficile.

Tutte le partecipanti raccomanderebbero il corso ai futuri studenti. Eccetto una studentessa che ritiene che il corso potrebbe essere migliorato con più traduzione e meno lettura e analisi grammaticale dei testi, tutte pensano che sia necessario aumentare il numero di ore di lezione.

\subsubsection{Analisi dei risultati del questionario}

Cercheremo ora di dare possibili spiegazioni alle risposte delle studentesse, analizzandole da due punti di vista: a) dalla posizione del ruolo e delle competenze esistenti e di quelle richieste a uno studente di italianistica che si incontra per la prima volta con una delle varietà della lingua; b) dalla posizione del ruolo e delle competenze dell'insegnante, prendendo in esame la struttura e la programmazione del sillabo del suddetto corso.

È possibile collegare la risposta alla prima domanda, con la quale abbiamo scoperto che le studentesse dispongono di esperienze molto ridotte nel campo dei linguaggi settoriali, con uno dei punti cardinali dell'organizzazione curricolare ovvero - quello della questione inerente al momento giusto per l'inserimento di un corso sui linguaggi settoriali. Concordiamo con chi ritiene che l'apprendimento e l'acquisizione, e di conseguenza una solida padronanza della lingua generale, dovrebbero precedere l'introduzione di un corso sui linguaggi settoriali (Freddi in Balboni, 2000: X-XI). Riteniamo che il lavoro dell'apprendente sarà agevolato e lui stesso sarà pronto ad avventurarsi nello studio dei linguaggi settoriali solo dopo aver assimilato i meccanismi di funzionamento dell'intero sistema linguistico e dopo aver acquisito le regole morfosintattiche e quelle dell'impostazione testuale. Questa nostra opinione deriva certamente da un chiaro e decisivo orientamento universitario nell'insegnamento delle lingue straniere che avvantaggia, anche in questa sede, l'ordine lingua generale $\left({ }^{\circledR}\right.$ linguaggi settoriali. Prendendo in considerazione l'apprendimento universitario delle LS, consideriamo che il momento opportuno per l'introduzione di un corso sui 
linguaggi settoriali sia nell'ambito dei corsi di laurea magistrale o durante il dottorato di ricerca (Drljević, 2016: 495).

Non sorprende il fatto che la maggioranza delle studentesse, come motivo principale per l'iscrizione al corso, indica la volontà di migliorare le competenze nella traduzione specializzata. Questa motivazione deriva dal fatto che il programma di studi quadriennale, seguendo un orientamento tradizionale degli studi filologici, prevede perlopiù un'approfondita analisi e la traduzione dei testi letterari e giornalistici, e molto meno quelli di carattere scientifico e settoriale. Dal punto di vista didattico e della programmazione del curricolo, il corso stesso e il suo approccio testuale e traduttivo rappresentano una buona e necessaria continuazione nello sviluppo e nel consolidamento della competenza traduttiva generale degli studenti. Ovviamente, non dobbiamo perdere di vista che per una buona parte delle studentesse, il bisogno di un ampliamento delle conoscenze non strettamente linguistiche è stato la principale motivazione per l'iscrizione al corso. Questa risposta, secondo la nostra opinione, è un indicatore importante dell'interesse degli studenti a seguire le richieste e i cambiamenti del mercato del lavoro.

La quasi totalità delle risposte sul buon andamento e sulla struttura adeguata del corso parlano chiaro non soltanto dell'impostazione didattica del corso, ma confermano anche la necessità di possedere una conoscenza solida della lingua generale prima di dedicarsi alle specificità linguistiche aggiuntive, quali appunto quelle di cui consiste un linguaggio settoriale.

Con un possibile aumento del numero di lezioni potremmo, prima di tutto, dedicarci con più attenzione ai singoli settori scientifico-professionali, ampliando le attività di analisi linguistica dei testi, di analisi lessicale e di creazione dei glossari, e infine della traduzione specializzata, seguendo sempre l'ordine didattico proposto nella parte 2.3.

\section{Considerazioni conclusive}

Abbiamo scelto di trattare il tema dei ruoli e competenze dell'insegnante e dello studente in un corso sui linguaggi settoriali di una lingua straniera perché ci interessava esaminare questo aspetto dal punto di vista di chi insegna e di chi studia, nell'ambito degli studi universitari filologici.

La nostra ricerca sulle competenze dell'insegnante è partita da due questioni: l'insegnante deve possedere solo elevate conoscenze 
Jelena R. Drljević

linguistiche? Oltre alle necessarie conoscenze linguistiche l'insegnante deve predisporre anche di conoscenze non linguistiche, ovvero concettuali, dei diversi settori scientifico-professionali? Prendendo in considerazione la formazione degli insegnanti che di solito si basa sullo studio universitario delle lingue straniere e su specializzazioni di orientamento umanistico, con successivo ampliamento dei propri ruoli, e quindi anche delle competenze richieste dal contesto dell'insegnamento dei linguaggi settoriali, abbiamo tratto una possibile conclusione sui doveri professionali che a tale categoria competono: l'insegnante di un corso sui linguaggi settoriali è un linguista che, grazie alle sviluppate competenze linguistiche, analizza la lingua dei testi appartenenti ai vari settori dell'attività umana. Inoltre, è auspicabile una certa dose di curiosità verso i contenuti non strettamente linguistici, che scoprirà insieme ai suoi studenti o con il sostegno degli esperti di un certo settore professionale.

Per lo studente, d'altra parte, il corso sui linguaggi settoriali può significare lo sviluppo di una vasta gamma di conoscenze linguistiche e non linguistiche, il cui raggiungimento viene stabilito all'inizio del corso, in base ad un'approfondita analisi dei bisogni. Tali opportunità variano: dallo sviluppo delle competenze linguistico-comunicative generali al rafforzamento degli aspetti importanti per un uso specifico della lingua (traduzione, formazione delle parole, creazione dei glossari, sviluppo di conoscenze non linguistiche ecc). Secondo i dati rilevati dal questionario che abbiamo distribuito agli studenti d'Italianistica dell'Università di Belgrado, la motivazione principale per l'iscrizione ad un corso sui linguaggi settoriali sta nel desiderio di sviluppare ulteriormente la competenza traduttiva - traducendo appunto testi specifici. Secondo la nostra opinione, il motivo di questa scelta sta nel fatto che gli studenti di orientamento linguistico, nonostante l'interdisciplinarità nell'apprendimento delle lingue straniere esistente nell'ultimo decennio, sono tradizionalmente legati alla traduzione, vista come uno dei metodi didattici nello studio della lingua ${ }^{8}$. La competenza traduttiva ormai solidamente sviluppata

8 Per maggiori dettagli sull'uso della traduzione come uno dei metodi didattici nell'apprendimento della lingua consultare Piletić, D., Drljević, J. (2015). Il ruolo della traduzione nell'insegnamento del lessico di lingua italiana come LS a livello universitario. U: Nikodinovska, R. (prir.), Atti del convegno internazionale Parallelismi linguistici, letterari e culturali - 55 anni di studi italiani, 469-481. Skopje: Edizione dell'Università "Ss. Cirilo e Metodio". 
INSEGNARE I LINGUAGGI SETTORIALI DI UNA LS A LIVELLO ...

nel corso degli studi quadriennali, nonché un'elevata conoscenza delle strutture morfosintattiche e lessicali della lingua, rappresentano il punto di forza degli studenti laureati in lingua e letteratura italiana. Inoltre, $\mathrm{i}$ nostri studenti, oltre ad essersi dedicati ai contenuti specializzati che gli interessavano in particolar modo, erano anche curiosi di conoscere molti altri settori con i loro linguaggi specifici.

Concludiamo che i ruoli e le competenze di entrambi i partecipanti a un corso sui linguaggi settoriali possono essere numerosissimi e rappresentano, a nostro avviso, una duplice opportunità: è possibile migliorare, da una parte, l'aspetto linguistico e comunicativo delle conoscenze già possedute $\mathrm{e}$, dall'altra, cominciare una specializzazione nei settori non linguistici, usando la lingua in modo veicolare.

\section{BIBLIOGRAFIA}

Altieri Biagi, M. L. (1990). L'avventura della mente. Studi sulla lingua scientifica. Napoli: Morano Editore.

Drljević, J. (2016). Insegnamento dei linguaggi settoriali negli studi filologici. Esperienza con gli apprendenti serbofoni d'italiano LS. In R. Scotti Jurić, N. Poropat Jeletić, E. Matticchio (ed), Studi filologici e interculturali tra traduzione e plurilinguismo (pp. 493-506). Roma: Aracne editrice int.le S.r.1.

Douglas, D. (2000). Assessing Languages for Specific Purposes. Cambridge: Cambridge University Press.

Dudley-Evans, T., St John, M. (1998). Developments in ESP: A multidisciplinary approach. Cambridge: CUP.

Đorović, D. (2015). Analiza potreba u nastavi stranog jezika struke. Beograd: Filozofski fakultet Univerziteta u Beogradu.

Gotti, M. (1998). La valutazione delle competenze linguistiche di carattere specialistico. In M. Pavesi, G. Bernini (ed), L'apprendimento linguistico all'università: le lingue speciali (pp. 227-245). Roma: Bulzoni Editore.

Gotti, M. (1992). Testi specialistici in corsi di lingue straniere. Firenze: La Nuova Italia.

Hutchinson, T., Waters, A. (1987). English for Specific Purposes. A Learningcentred Approach. Cambridge: Cambridge University Press. 
Jelena R. Drljević

Mazzotta, P., Salmon, L. (2007). Tradurre le microlingue scientificoprofessionali. Riflessioni teoriche e proposte didattiche. Torino: Utet Libreria.

Munby, J. (19789. Communicative Syllabus Design. Cambridge: Cambridge University Press.

Richterich, R., Chancerel, J. L. (1977). Identifying the Needs of Adults Learning a Foreign Language. Oxford: Pergamon Press.

Samardžić, M. (2006). Od rečenice do teksta. Uvod u sintaksu italijanske složene rečenice. Podgorica: Filozofski fakultet, Univerzitet Crne Gore.

Strevens, P. (1980). Teaching English as an international language: From practice to principle. Oxford: Pergamon Press.

Ewer, J. R. (1983). Teacher Training for EST: Problems and Methods. The ESP Journal, II (1), 9-31.

Tarone, E., Yule, G. (1989). Focus on the Language Learner. Oxford: Oxford University Press.

Wilkins, D. A. (1976). National syllabuses. Oxford. Oxford University Press.

\title{
Jelena R. Drljević
}

\section{NASTAVA STRANOG JEZIKA STRUKE NA UNIVERZITETU (SLUČAJ ITALIJANSKOG JEZIKA). ULOGA I KOMPETENCIJE NASTAVNIKA I STUDENATA}

\begin{abstract}
Sažetak
U radu smo ispitali uloge i kompetencije nastavnika i studenata kursa stranog jezika struke (u našem slučaju italijanskog) koji za cilj ima učenje i usvajanje specifične dimenzije jezika, upravo one koja je u vezi sa iskazivanjem određenih, stručnih znanja. Ulogu i očekivana znanja i nastavnika i studenata posmatrali smo i analizirali na osnovu konteksta izvođenja kursa - univerziteta - i u skladu sa didaktičkim ciljevima koje takav kontekst nameće.

Kada je reč o kompetencijama nastavnika, analizirali smo dva stava koja se pojavljuju u literaturi koja se bavi ovom temom (Gotti, 1992: 286-289): a) nastavnik mora posedovati, pored odličnih jezičkih znanja, i znanja koja se odnose na specifične pojmove neke stručne oblasti; b) u slučaju da nastavnik raspolaže isključivo jezičkim znanjima, trebalo bi da primeni didaktiku čiji je cilj razmena nejezičkih znanja sa studentima, i to kroz raznovrsne komunikativne aktivnosti. $\mathrm{Na}$ taj način bi i nastavnik i studenti bili u stanju da analiziraju i aspekat sadržaja nastavnog materijala.
\end{abstract}


Razvoj i stanje očekivanih jezičkih kompetencija studenata ispitali smo u odnosu na potrebe samih studenata. Predstavili smo didaktičku strukturu kursa Italijanski jezik struke, održanom u prvom semestru akademske. 2017/2018. na Katedri za italijanistiku Filološkog fakulteta Univerziteta u Beogradu. Polaznici kursa bili su studenti ove katedre, a kurs se, pored predstavljanja teorijskih saznanja sastojao i od praktičnog dela u okviru kog smo analizirali i pismeno prevodili stručne tekstove (ekonomske, pravne, političke, religijske) sa italijanskog na srpski jezik.

\section{QUESTIONARIO PER GLI STUDENTI DEL CORSO LINGUAGGI SETTORIALI DELL'ITALIANO, A.A. 2017/2018}

Il presente questionario è stato creato allo scopo di raccogliere dati rilevanti per una ricerca scientifica inerente al campo dei linguaggi settoriali e del loro apprendimento. Il questionario è anonimo. Vi preghiamo di rispondere alle domande cerchiando UNA delle opzioni offerte:

1. Prima di iscriverti al corso Linguaggi settoriali dell'italiano, quanta esperienza avevi con la lettura, l'analisi e la traduzione di testi appartenenti ad un settore scientifico-professionale?

\section{a) Nessuna}

b) Poca (solo lettura, nessuna traduzione del testo)

c) Abbastanza (specificare i campi scientifico-professionali ai quali appartenevano i testi che avevi letto e le attività svolte sui testi - analisi, traduzione)

\section{Quale delle seguenti descrive meglio la ragione d'iscrizione a questo corso?}

a) Conoscenza dei termini specifici inerenti ad uno o più campi scientificoprofessionali

b) Sviluppo dell'abilità di comprensione scritta (lettura) delle diverse tipologie testuali inerenti ad uno o più campi scientifico-professionali

c) Sviluppo delle competenze traduttive nell'ambito della traduzione dei testi specifici

d) Ampliamento delle conoscenze generali linguistico-comunicative della lingua italiana

e) Consolidamento delle conoscenze linguistiche e non-linguistiche utili per il futuro inserimento nel mercato del lavoro 
Jelena R. Drljević

3. La difficoltà del corso è stata in linea con le tue aspettative?

a) Sì, ho seguito senza problemi l'intero corso

b) Abbastanza (sottolineare il problema nel seguire il corso: i testi generalmente troppo difficili, la terminologia dei testi troppo difficile, la struttura del corso inadeguata, i compiti poco motivanti)

c) No (sottolineare: l'intero corso è stato troppo difficile/l'intero corso è stato troppo facile)

4. Raccomanderesti questo corso ad altri studenti?
a) Sì
b) No
5. Se no, perché?

6. In quali modi questo corso potrebbe essere migliorato?

a) Più ore di lezione

b) Più lettura e analisi grammaticale dei testi, meno traduzione

c) Più traduzione, meno lettura e analisi grammaticale dei testi

d) Altro: 